\title{
Effect of Chemical Stabilizers in Silver Nanoparticle Suspensions on Nanotoxicity
}

\author{
Eunjoo Bae, Hee-Jin Park, Junsu Park, Jeyong Yoon, Younghun Kim, ${ }^{\dagger}$ Kyunghee Choi, ${ }^{\ddagger}$ and Jongheop Yi* \\ World Class University (WCU) Program of Chemical Convergence for Energy \& Environment $\left(C_{2} E_{2}\right)$, \\ School of Chemical and Biological Engineering, College of Engineering, Seoul National University (SNU), \\ Seoul 151-742, Korea.*E-mail: jyi@snu.ac.kr \\ ${ }^{\dagger}$ Department of Chemical Engineering, Kwangwoon University, Seoul 139-701, Korea \\ "National Institute of Environmental Research, Incheon 404-708, Korea \\ Received June 15, 2010, Accepted December 9, 2010
}

\begin{abstract}
Colloidal silver nanoparticles (AgNPs) have been commercialized as the typically stabilized form via the addition of a variety of surfactants or polymers. Herein, to examine the effects of stabilizing AgNPs in suspension, we modified the surface of bare AgNPs with four type of surfactants (NaDDBS, SDS, TW80, CTAB) and polymers (PVP, PAA, PAH, CMC). The modified AgNPs was applied to compare suspension stability and nanotoxicity test using Escherichia coli (E. coli) as a model organism. Modification of AgNPs surface using chemical stabilizer may be not related with molecular weight, but chemical structure such as ionic state and functional group of stabilizer. In this study, it is noteworthy that AgNPs modified with a cationic stabilizer $(\mathrm{CTAB}, \mathrm{PAH})$ were importantly toxic to $E$. coli, rather than anionic stabilizers (NaDDBS, SDS). Comparing similar anionic stabilizer, i.e., NaDDBS and SDS, the result showed that lipophilicity of chemical structure can affect on E. coli, because NaDDBS, which contains a lipophilic benzene ring, accelerated the cytotoxicity of AgNPs. Interestingly, none of the stabilizers tested, including biocompatible nonionic stabilizers (i.e., TW80 and cellulose) caused a reduction in AgNP toxicity. This showed that toxicity of AgNPs cannot be reduced using stabilizers.
\end{abstract}

Key Words: Agglomeration, Suspension stabilizer, Silver nanoparticle, Escherichia coli

\section{Introduction}

Silver nanoparticles (AgNPs) have expanded commercially in a variety of fields, including textiles, cosmetics, spray cleaning agents, antipyretic, plastics, and paints. The AgNPs are mostly modified to improve stability of inherent nano-property. However, the AgNP itself is classified as an environmental hazard, because it releases silver ions and absorps on a biological organ. ${ }^{1}$ Therefore, the application of AgNPs-binding stabilizers, due to improperly gathered data, has recently lead a controversy about whether it can accelerate nanotoxicity of bare AgNPs or not. In addition, surface modification of AgNPs as newly issued factor in nanotoxicity researches. Importantly, change of surface property can affect on the mechanism between AgNPs and biological organs. From the point of view, the use of stabilizer-capped AgNPs is an important environmental issue as a matter of urgency.

Surface chemistry can be controlled by parameters such as molecular weight, charge, chemical functionality, and hydrophilicity, which plays an important role in determining the penetration of nanoparticles into a cell. Alkilany et al. ${ }^{16}$ reported that the apparent cytotoxicity of gold nanorods is caused by presence of free $\mathrm{CTAB}$, and not CTAB that is actually associated with gold nanorods, because serum proteins from the biological media protect CTAB-capped gold nanorods against similar charges. Chemical with heavy molecular weight as a toxicity parameter was determined to be assumed to reduce nanotoxicity because of its coating effects of toxic core material releasing. Among these parameters, electrostatic interactions may lead to membrane disruption. However, polycationic polymers appear to play a role in cell-penetrating peptide, not protect toxic core nanomaterial. Although the surface chemistry of AgNPs, is clearly an important issue available information on the relationship between chemistry and toxicity/suspension stability is limited at present.

Nanoscale effects are based on maintaining nanosized nanoparticles in a suspension. A variety of chemicals to be used in to stabilize suspensions of AgNPs. To achieve this, dispersing agents, such as surfactants, polymers, and reducing agents are used in the aqueous phase. The following chemicals have been mostly used to suspend AgNPs in an aqueous phase; stabilizers ${ }^{2-9}$ such as poly(acrylamide) (PAA), poly(vinyl alcohol) (PVA), poly(vinyl pyrrolidone) (PVP), poly(ethylene glycol) (PEG), 2-mercaptobenzimidazole, and multilayered polyelectrolyt$\mathrm{es}^{10,11}$ such as polyalkylamine hydrochloride (PAH); surfac$\operatorname{tants}^{12-15}$ such as an aerosol form of sodium bis(2-ethyl-hexyl)sulfosuccinate, sodium dodecyl sulfate (SDS), and cetyltrimethylammonium bromide (CTAB). Among these stabilizers, we selected some to explain the mechanism of nanotoxicity due to change of surface according to surface chemistry as mentioned above.

From the standpoint of comparing AgNP and its stabilizer, this study examined with reference to 1) inherent toxicity; 2) ability to accelerate AgNPs toxicity; 3) their effect on toxicity; 4) reducing the toxicity of AgNPs. Among the numerous stabilizers that are used for improvement of AgNPs suspension, a series of surfactants and polymers were selected for study, based on their molecular structure and charge, since they are considered to be the major cause in changing the surface chemistry of AgNPs. The stability of suspensions of bare AgNPs changes when different stabilizers such as surfactants and polymers, which are used to control the shape/size of nanoparticles, are 
used. $^{17,18}$ The compounds selected were cationic materials (CTAB and PAH) and anionic materials (NaDDBS, SDS, and PAA). In addition, sodium dodecylbenzenesulfonate (NaDDBS), a surfactant with a higher hydrophile-lipophile balance (HLB) was examined, since it can serve as a measure of the relative contributions of the hydrophilic and lipophilic regions of a molecule, as opposed to SDS. The characteristics of NaDDBS are similar to those for SDS from the standpoint of molecular structure and anionic charge, but additional functional group (such as benzyl ring) present. In additional, biocompatible compounds such as polyoxyethylenesorbitanmonolaurate (TWEEN 80 ) and carboxy methyl cellulose (CMC), which are typically used as dispersing substances in medicinal and food products, were used to investigate methods for reducing the toxicity of AgNPs.

Escherichia coli (E. coli), a gram negative bacterium, was used as a conceptual model for environmental exposure. Bacteria have the ability to grow in a variety of harsh environments. In natural environments, bacteria are frequently challenged by nutrient starvation and other stresses. Recently, E. coli has been researched for nanotoxicity in the environment.

\section{Experimental}

An AgNP solution was prepared using powdered, 99\% pure silver (Sigma-Aldrich), with a particle size $<150 \mathrm{~nm}$. Tetrahydrofuran (THF, 99.8\%; Fluka) was used to suspend the AgNPs in solution. Surfactants such as NaDDBS (Sigma-Aldrich), SDS (96\%, Fluka), TWEEN 80 (Sigma-Aldrich), CTAB (SigmaAldrich) were used. In addition, PVP (Sigma-Aldrich), PAA (Sigma-Aldrich), PAH (Sigma-Aldrich), CMC (Sigma-Aldrich) were used as polymer stabilizers. Details of the properties of these materials, such as molecular weight, HLB, and charge type are shown in Table 1.

Bare AgNPs suspensions were prepared using physical methods, i.e., sonication, stirring, and nanofilteration from commercial Ag nanopowder. The AgNPs were stirred for 1 day. Most of the $\mathrm{Ag}^{+}$ions from the silver nanopowder produced during sonication and stirring were removed using a potentiostatic method with a three electrode-electrochemical cell (silver wire as a pseudo-reference electrode and a platinum plate as a working electrode, respectively). The total Ag concentration was determined by inductively coupled plasma spectrometry (ICP; Shimadzu, ICPS 7500). The average hydrodynamic diameter of AgNPs was determined to be $50 \mathrm{~nm}$ by dynamic light scattering (DLS; Otsuka, ELS-8000). The AgNPs were mixed with surfactant $(0.1-10 \mathrm{mM})$ and polymer $(0.001-0.1 \mathrm{mM})$ stabilizers. Agglomeration occurred with in 1 day after the AgNPs were mixed with the stabilizers and the supernatant solution was removed by centrifugation. All samples were stored in a dark room and maintained at a temperature below $25^{\circ} \mathrm{C}$.

AgNPs suspensions were analyzed by high-resolution transmission electron microscopy (HR-TEM; $300 \mathrm{kV}$, JEOL, JEM3010). The stability of suspensions of AgNPs mixed with stabilizers was determined by measuring the diffusion coefficient, which represents the amount of diffusion of AgNPs per unit time. These measurements were made by the DLS method. Surface charges of bare AgNPs and stabilizer-capped AgNPs were determined by electrophoretic light scattering (ELS; Otsuka, ELS-8000).

Escherichia coli DH5 $\alpha$ was used as an indicator bacterium for evaluating the toxicity of stabilizer-capped AgNPs. A single colony of E. coli was inoculated with an AgNPs sample in LuriaBertani (LB) broth media, and then incubated at $37^{\circ} \mathrm{C}$ for $18 \mathrm{hr}$. The cells were collected by centrifugation at $1000 \times \mathrm{g}$ for $10 \mathrm{~min}$ and washed three times with deionized water (Barnsted NANO Pure, USA) to remove all soluble components. An approximate population of $2 \times 10^{6} \mathrm{CFU}$ (colony forming units) $/ \mathrm{mL} E$. coli cells was exposed to a $1 \mathrm{mg} / \mathrm{L}$ suspension of each AgNPs sample in $10 \mathrm{mM}$ phosphate buffer ( $\mathrm{pH} \mathrm{7.1)}$ for $3 \mathrm{hr}$. The surviving population of $E$. coli cells was determined by the spread plate method $(\mathrm{CFU} / \mathrm{mL})$.

\section{Results and Discussion}

Stability of AgNP suspensions are shown in Fig. 1. Change of diffusion coefficient of surface-modified AgNPs to compare bare AgNPs $\left(\mathrm{D}_{0}\right)$ was used. Increase of diffusion coefficient indicated the improvement of suspension stability, i.e., effects of stabilizer. In Fig. 1 (a-d), anionic surfactants, NaDDBS and SDS, were more effective in improving the stability of the AgNPs suspensions, rather than nonionic surfactants (TWEEN

Table 1. Data for the chemical stabilizers used in this study.

\begin{tabular}{cllcl}
\hline Group & Stabilizer & \multicolumn{1}{c}{ Normal use } & Molecular weight, g/mol & Chemical structure \\
\hline \multirow{3}{*}{ I } & NaDDBS & Anionic Surfactant & 348 & $\mathrm{C}_{18} \mathrm{H}_{29} \mathrm{SO}_{3} \mathrm{Na}$ \\
& SDS & Anionic Surfactant & 288 & $\mathrm{C}_{12} \mathrm{H}_{25} \mathrm{SO}_{4} \mathrm{Na}$ \\
& TW80 & Nonionic Surfactant & 1,310 & $\mathrm{C}_{64} \mathrm{H}_{124} \mathrm{O}_{26}$ \\
& CTAB & Cationic Surfactant & 365 & $\mathrm{C}_{19} \mathrm{H}_{42} \mathrm{BrN}$ \\
\hline & PVP & Nonionic Polymer & $40,000^{*}$ & $\left(\mathrm{C}_{6} \mathrm{H}_{9} \mathrm{NO}\right)_{\mathrm{n}}$ \\
& PAA & Anionic Polymer & $15,000^{*}$ & $\left(\mathrm{C}_{3} \mathrm{H}_{3} \mathrm{NaO}_{2}\right)_{\mathrm{n}}$ \\
& PAH & Cationic Polymer & $15,000^{*}$ & $\left(\mathrm{C}_{3} \mathrm{H}_{8} \mathrm{ClN}_{\mathrm{n}}\right.$ \\
& CMC & Cellulose & $90,000^{*}$ & $\left(\mathrm{C}_{28} \mathrm{H}_{30} \mathrm{Na}_{8} \mathrm{O}_{27}\right)_{\mathrm{n}}$ \\
\hline
\end{tabular}

Note: NaDDBS, sodium dedecylbenzenesulfonate Na salt; SDS, Sodium dodecyl sulfate; TW80, Polysorbate 80; CTAB, CetylTrimethyl Ammonium Bromide; PVP, Polyvinylpyrrolidone; PAA, Polyacrylic Acid; PAH, Poly (allylamine hydrochloride); CMC, Carboxymethyl cellulose *: average molecular weight. 

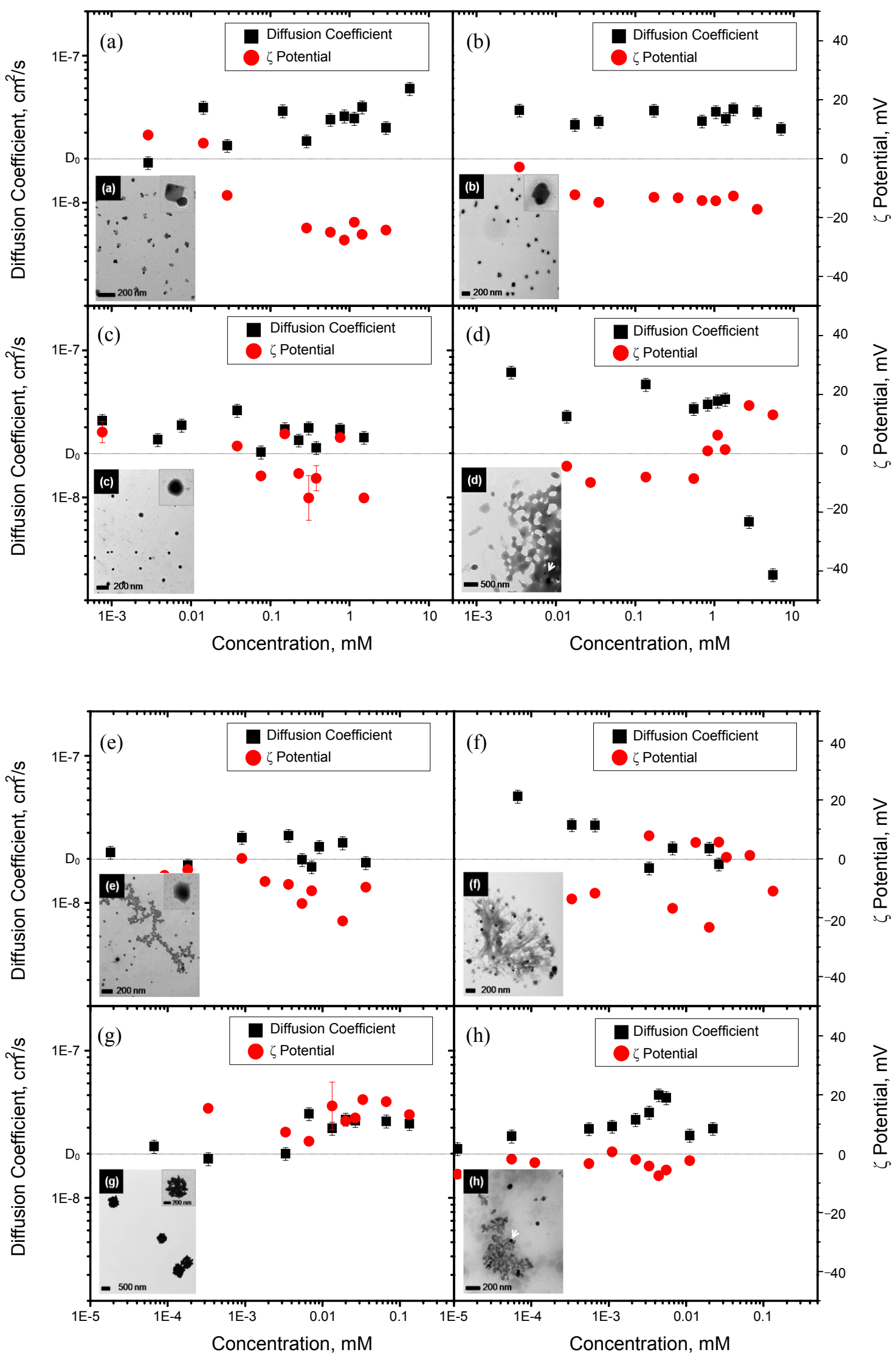

Figure 1. Diffusion coefficients, zeta potentials, and TEM images of AgNPs coated with Group I (a) NaDDBS, (b) SDS, (c) TW80, and (d) CTAB; Group II (e) PVP, (f) PAA, (g) PAH, and (h) CMC. (TEM images showed at $10 \mathrm{mM}(\mathrm{a}-\mathrm{d})$ and $0.1 \mathrm{mM}(\mathrm{e}-\mathrm{h})$ ), $\mathrm{D}_{0}$ : initial diffusion coefficient). 

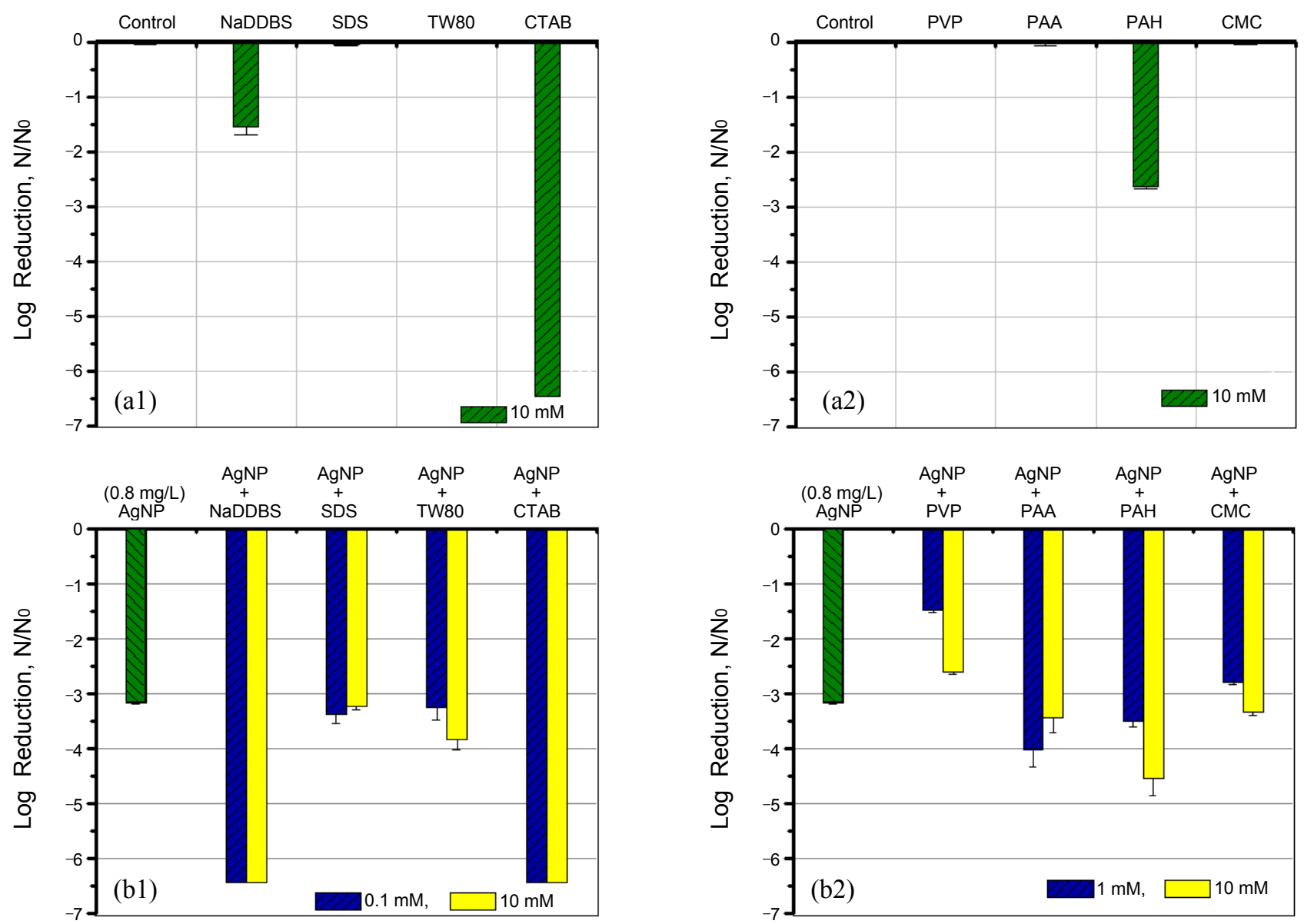

Figure 2. Inactivation of E. coli by (a) stabilizer only and (b) mixture of AgNPs and stabilizer.

80, Fig. 1c) and a cationic surfactant (CTAB, Fig. 1d). Otherwise, the use of CTAB resulted in an increase in the diffusion coefficients, following by a dramatic decrease at diameters of $>1 \mathrm{mM}$. At overdosage $(0.1 \mathrm{mM}$ concentrations ranges $)$ of cationic surfactant can carry out decreasing suspension stability. The reason is the change of the surface charge of the AgNPs from a negative to a positive charge. In TEM images (Fig. 1d), the AgNPs solutions had a milky appearance and the AgNPs were mixed with surfactant material. Otherwise, the nonionic surfactant had no effect on the surface charge of the AgNPs and the zeta potentials fluctuated between $0 \mathrm{mV}$ and a negative $30 \mathrm{mV}$. TEM images of AgNPs suspensions with nonionic surfactants indicate that they were well-dispersed on a single AgNP.

The suspension stability of AgNPs, coated with polymer stabilizer, was improved, but not better than surfactant effects. The lower stability conferred by a polymer is related to the higher viscosity of the polymer compared to that of a surfactant in the Stokes-Einstein equation. From this point of view, the diffusion coefficients of AgNPs coated with PAA also decreased with increasing concentrations of PAA (Fig. 1f). The addition of PVP, PAH, and CMC did not importantly increase the diffusion coefficient compared to bare AgNPs ( $\mathrm{D}_{0}$, Fig. 1e, 1g, and1h). The surface charges of the AgNPs were changed in a positive direction in the presence of PAH (Fig. 1h). PAH polyelectrolytes were successfully adsorbed onto the surface of the AgNPs, ${ }^{11}$ resulting in the formation of microsized colloids. In TEM images, AgNPs formed new types of agglomerates in which several particles participated via interactions with PAH. However, the addition of an anionic polymer, PAA, resulted in the surface charge fluctuating between 0 and negative $20 \mathrm{mV}$ and CMC had no effect on the surface charge. The TEM images showed that AgNPs were present in the form of cluster or mixture with PVP, PAA, and CMC.

The results of suspension stability of AgNPs show that surfactant may be helpful to improve suspension rather than polymer stabilizer. In addition, surface charge of AgNPs was changed by cationic surfactant and polymer from negative to positive. Otherwise, anionic and nonionic stabilizer did not change the surface charge.

The inactivation of E. coli was shown in Fig. 2. The same parameter such as surface charge type, HLB, molecular weight of stabilizers was used with suspension tests. Cationic stabilizers are used extensively to disrupt cell membranes and transport materials into cells. The results showed that when the AgNPs were coated with cationic organic materials (CTAB and PAH), the surface charge of the AgNPs became positive, resulting in the lysis of E. coli cells (Fig. 3d and 3g). Cationic charged AgNPs showed the highest lethal effects (Fig. $2 \mathrm{~b} 1$ and 2b2), probably because the cell membrane is negatively charged and may enter into electrostatic interactions with the positively charged AgNPs. 

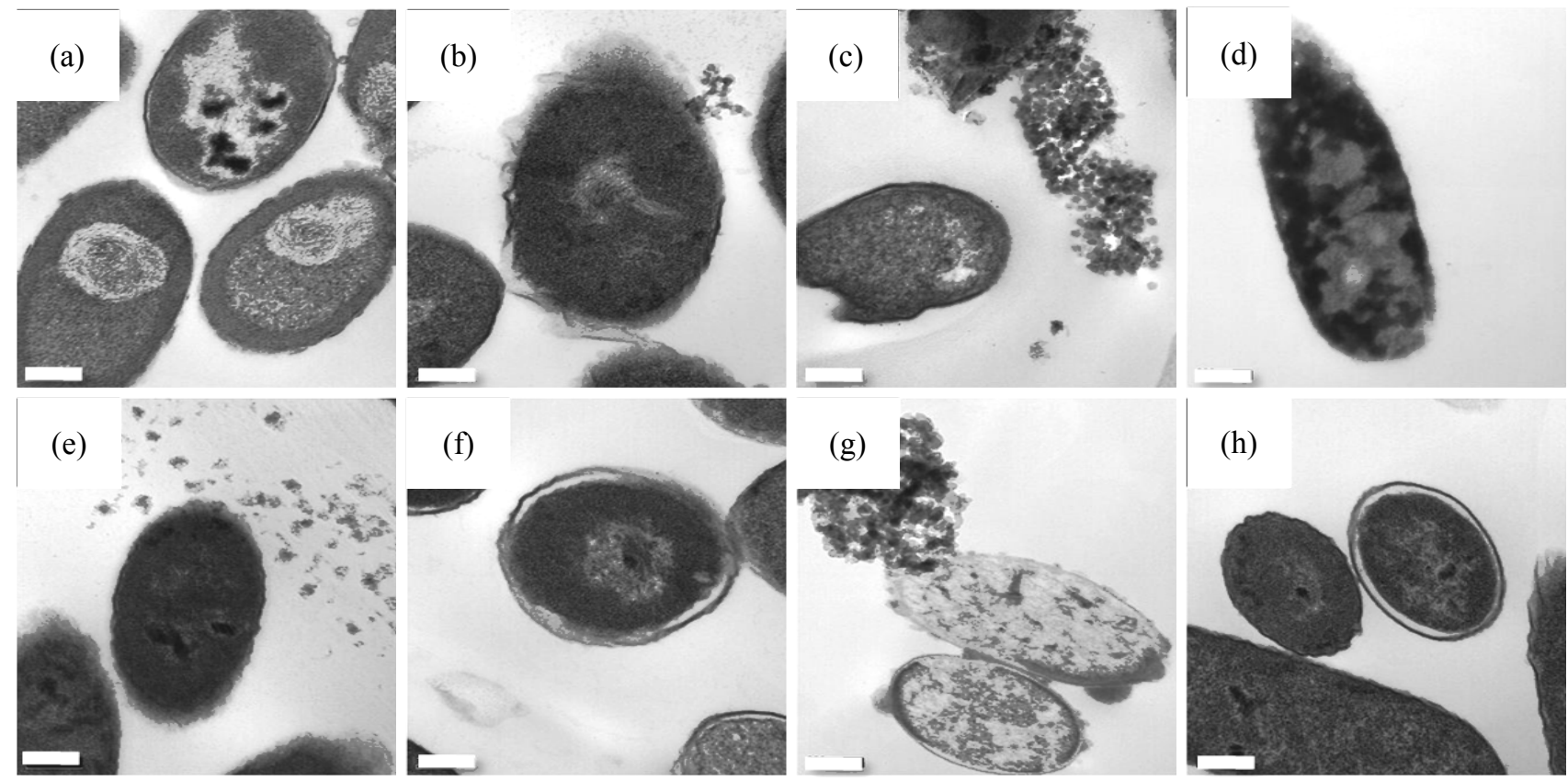

Figure 3. TEM images of AgNPs agglomerated with chemical stabilizers; Group I. (a) NaDDBS, (b) SDS, (c) TW80, and (d) CTAB; Group II. (e) PVP, (f) PAA, (g) PAH, and (h) CMC (scale bar $=200 \mathrm{~nm}$ ).
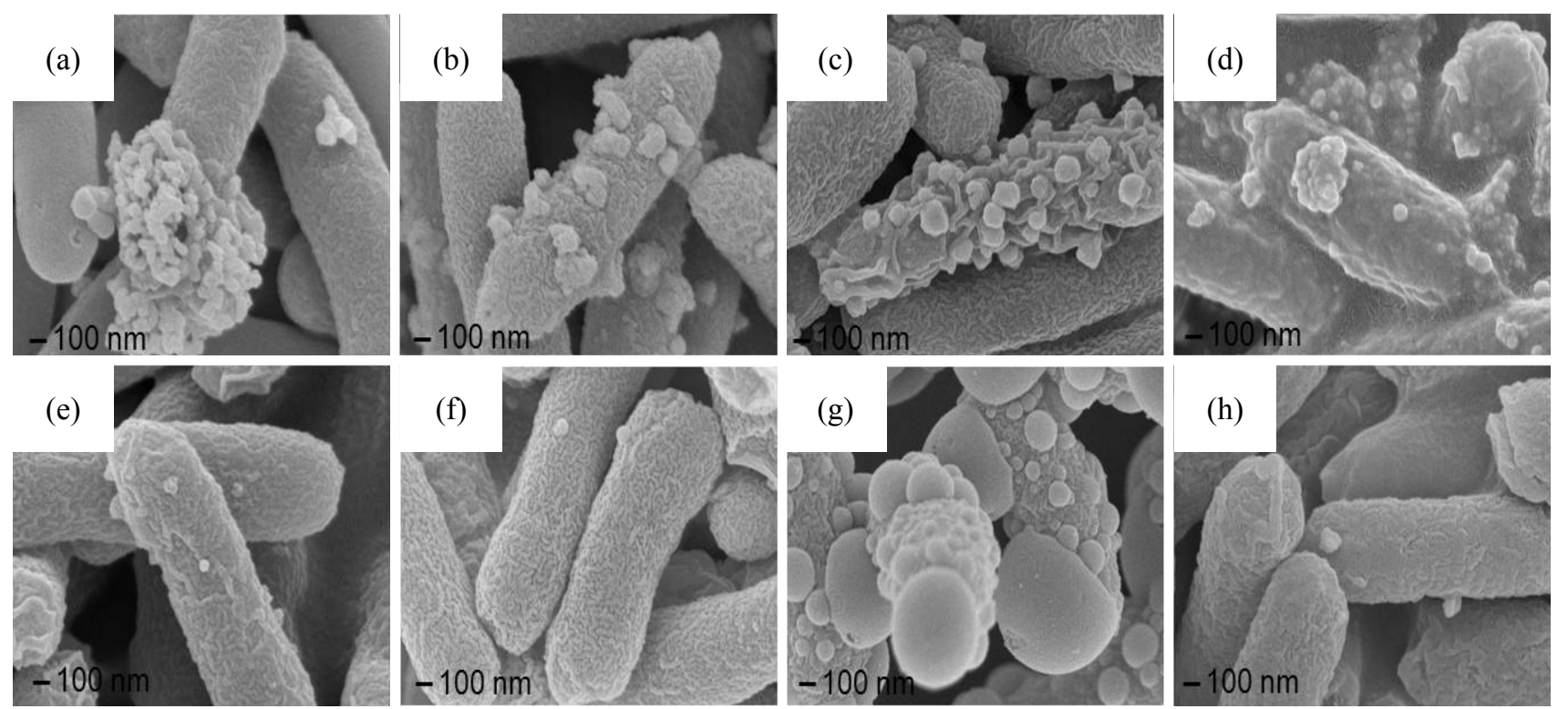

Figure 4. SEM images of AgNPs agglomerated with chemical stabilizers; Group I. (a) NaDDBS, (b) SDS, (c) TW80, and (d) CTAB; Group II. (e) PVP, (f) PAA, (g) PAH, and (h) CMC.

Both cationic stabilizers had a strong inherent toxicity and inactivated E. coli. In addition, the cationic surfactant (CTAB) showed a lethal bactericidal action, even at low concentrations $(0.03 \mathrm{mM})$. Alkilany et al. ${ }^{16}$ reported that low toxic Au nanoparticle as core materials show toxicity only in case when free CTAB is released from CTAB-capped Au nanoparticles. In the present study, the toxicity of PAH-coated AgNPs (at 0.06 $\mathrm{mM}$ PAH) was accelerated compared to the toxicity of bare AgNPs and PAH. This result is not in agreement with the report that PAH-overcoated gold nanorods are relatively nontoxic and that the cytotoxicity of nanoparticles is not correlated with its surface charge. ${ }^{16}$ The fact that serum was used in the case of gold nanorods and the nature of the core material (gold $v s$. silver) may explain this discrepancy.

The results for anionic stabilizers (NaDDBS, SDS, and PAA) and nonionic stabilizers (TW80, PVP, and CMC) were complicated and were not dependent on the surface charge in inactivating E. coli. AgNPs stabilized with SDS showed no acceleration 
in antibacterial activity. Cho et al. ${ }^{19}$ concluded that the negative surface charge of SDS interferes with the absorption of microbial cells to the surface of AgNPs or Ag ions by electrostatic effects. However, based on the findings of this study, we conclude that the HLB of anionic surfactants plays a more important role in interactions with the cell membrane than the negative charge properties. NaDDBS and SDS are both anionic surfactants, but the difference is that NaDDBS contains a benzene ring in its structure, resulting in a lower HLB number than that of SDS. The higher lipophilicity of NaDDBS induced unstable fluctuating diffusion coefficients with concentration rather than those for SDS-coated AgNPs (Fig. 1a and 1b). The use of an anionic surfactant itself may lead to denaturation and the loss of biological activity because it binds strongly to proteins. ${ }^{20}$ The higher lipophilicity of NaDDBS may increase the extent of interactions of cell membrane components such as lipopolysaccharides, resulting in an acceleration of toxic effects. It was also reported that subnanometric gold particles with alternating anionic and hydrophobic groups readily penetrated the plasma membrane without disrupting the lipid bilayer. Otherwise, hydrophilic-functional groups such as -OH (TW80) and -COOH (CMC) indicate that TW80- and CMC-coated AgNPs did not contribute to the toxicity AgNPs. Hoshino et al. ${ }^{21}$ reported that the properties of QDs are not related to those of QD-core materials but to the molecular covering of the surface of QDs. However, the present study showed that charge properties are more significant than functional group types.

In this study, charged AgNPs (such as CTAB- and PAH-coated) induced the transient poration of $E$. coli membranes to enter the cells, a process associated with cytotoxicity. CTAB, which is used in the synthesis, has membrane-compromising properties with a poor biocompatibility. Au nanorod is almost nanotoxic. However, the toxicity of CTAB-capped Au nanorods is increased by the presence of a coating polymer such as poly (diallyldimethylammonium chloride)-poly (4-styrenesulfonic acid) and polystyrenesulfonate. The present study had expected to observe surface coating effects by polymers such as CMC. However, in most cases when chemical stabilizers were used, the toxicity of the AgNPs was increased than bare AgNPs. The increase in the toxicity of AgNPs can be attributed to potential reactions between AgNPs (or residual Ag ions) and chemical stabilizers used. Silver ions are release from AgNPs coated with polymers such as PVP and PVA.

The different stabilizers of interactions between gram-negative bacteria and AgNPs are illustrated in the TEM images (Fig. 3) and SEM images (Fig. 4). Unlike $\mathrm{Ag}^{+}$ions and bare AgNPs, cells exposed to AgNPs stabilized with chemicals underwent instant cell disruption and the cytoplasm tends to spill out of the cell. Significant morphological changes occurred in cases of the addition of a cationic surfactant (CTAB) and a cationic polymer (PAH). AgNPs coated with cationic stabilizers resulted in perforated membranes and subsequent cell lysis (Fig. 3d, 3g; Fig. 4d, 4g). PAH-coated AgNPs had a strong effect on $E$. coli and most of the cytoplasm was lost (Fig. 3g). CTABcoated AgNPs also adversely affected $E$. coli, resulting in the loss or aggregation of cytoplasm (Fig. 3d).

Some condensed substances were clearly visible in the center of cells that had been treated with AgNPs with anionic and nonionic stabilizers (Fig. 3a, 3b, 3e, 3f, and 3h). The structures were twisted and aggregated together in the center of the cell. This cytoplasm transformation was observed more often in NaDDBS-coated AgNPs than the others (Fig. 3a; Fig. 4a). Detachment between the cytoplasm and the cell wall was observed in cases of AgNPs that had been coated with NaDDBS, SDS, PAA, and CMC. Partial loss of the cell membrane was observed in $E$. coli that had been exposed to AgNPs coated with all of the anionic and nonionic stabilizers.

\section{Conclusions}

In conclusion, the inactivation of $E$. coli was not correlated with the properties of neutral stabilizers (TW80, PVP, and $\mathrm{CMC}$ ), but, rather, to ionic type and hydro/lipophilicity. Diffusion coefficient data indicate that surfactants were more effective instabilizing a suspension of AgNPs than polymers. Suspension stability improved mobility in environmental exposure. Surface chemistry reactivity was not correlated with suspension stability, but, rather, with cationic properties and hydrophobicity. The findings demonstrate that the toxicity of AgNPs is accelerated in cases of stabilizers with cationic properties and hydrophobicity, but was not reduced by biocompatible compounds or polymers. This suggests that the preferred use of AgNPs is in the field of biocidal or antiseptic agents, but not in biomedicine. Importantly, when AgNPs coated with stabilizers are released into the environment, toxicity may be sustained when they have an improved suspension stability. The disposal of AgNPs should be strictly controlled to prevent incidents of accidental toxicity in the environment.

Acknowledgments. This work was supported by the EcoTechnopia 21 project of Ministry of Environment (Korea) [grant number 079-071-055]. Authors appreciate WCU (World Class University) program through the National Research Foundation of Korea funded by the Ministry of Education, Science and Technology (R31-10013).

\section{References}

1. Muller, N. C.; Nowack, B. Environ. Sci. Technol. 2008, $42,4447$.

2. Chen, D.; Huang, Y. J. Colloid Interf. Sci. 2005, 255, 299.

3. Khanna, P. K.; Singh, N.; Kulkarni, D.; Deshmukh, S.; Charan, S.; Adhyapak, P. V. Mater. Lett. 2007, 61, 3366.

4. Mbhele, Z. H.; Salemane, M. G.; van Stittert, C. G. C. E.; Nedeljković, J. M.; Djoković, V.; Luyt, A. S. Chem. Mater. 2003, 15, 5019.

5. Hsu, S. L.; Wu, R. Mater. Lett. 2007, 61, 3719.

6. Kai, Z.; Qiang, F.; Jinghui, F.; Dehui, A. Mater. Lett. 2005, 59, 3682 .

7. Khanna, P. K.; Singh, N.; Kulkarni, D.; Deshmukh, S.; Charan, S.; Adhyapak, P. V. Mater. Lett. 2007, 61, 3366.

8. Popa, M.; Pradell, T.; Crespo, D.; Calderón-Moreno, J. M. Colloid. Surf. A 2007, 303, 184.

9. Tan, Y.; Jiang, L.; Zhu, D. J. Phys. Chem. B 2002, 106, 3131.

10. Logar, M.; Jančar, B.; Suvorov, D.; Kostanjšek, R. Nanotech. 2007, $18,325601$.

11. Radziuk, D.; Skirtach, A.; Sukhorukov, G.; Shchukin, D.; Möhwald, H. Macromol. Rapid Commun. 2007, 28, 848.

12. Prasad, B. L. V.; Arumugam, S. K.; Bala, T.; Sastry, M. Langmuir $\mathbf{2 0 0 5}, 21,822$. 
13. Chaudhari, V. R.; Haram, S. K.; Kulshreshtha, S. K.; Bellare, J. R.; Hassan,P. A. Colloids Surfaces A 2007, 301, 475.

14. Wei, G.; Wang, L.; Zhou, H.; Liu, Z.; Song, Y.; Li, Z. Appl. Surf. Sci. 2005, 252, 1189.

15. Sui, Z. M.; Chen, X.; Wang, L. Y.; Xu, L. M.; Zhuang, W. C.; Chai, Y. C.; Yang, C. J. Physica. E 2006, 33, 308.

16. Alkilany, A. M.; Nagaria, P. K.; Hexel, C. R.; Shaw, T. J.; Murphy, C. J.; Wyatt, M. D. Small 2009, 5, 701.

17. Wang, A. L.; Yin, H. B.; Ren, M.; Cheng, X. N.; Zhou, Q. F.;
Zhang, X. F. Acta Metallugica Sinica 2006, 19, 362.

18. Zhang, W.; Qiao, X. J. Chen, Mater. Sci. Eng. B 2007, 142, 1.

19. Cho, K.-H.; Park, J.-E.; Osaka, T.; Park, S.-G. Electrochimica Acta 2005, 51, 956.

20. Gloxhuber, C.; Künstler, K. Anionic Surfactants: Biochemistry, Toxicology, Dermatology, 2nd ed.; Marcel Dekker: New York, USA, 1992; p 27.

21. Hoshino, A.; Fujioka, K.; Oku, T.; Suga, M.; Sasaki, Y. F.; Ohta, T. NanoLett. 2004, 4, 2163. 